\title{
Production of biofuels by 5-hydroxymethylfurfural etherification using ion-exchange resins as solid acid catalysts ${ }^{+}$
}

\author{
Benjamín Torres Olea 1, Inmaculada Fúñez Nuñez 1, Cristina García Sancho 1, *, Juan Antonio \\ Cecilia ${ }^{1}$, Ramón Moreno Tost ${ }^{1}$ and Pedro Maireles Torres ${ }^{1}$ \\ 1 Departamento de Química Inorgánica, Cristalografía y Mineralogía (Unidad Asociada al ICP-CSIC) \\ Facultad de Ciencias, Universidad de Málaga, Campus de Teatinos, 29071 Málaga, Spain; \\ benjamin@uma.es (B.T.O.); inmafn@uma.es (I.F.N.); jacecilia@uma.es (J.A.C.), rmtost@uma.es (R.M.T.) and \\ maireles@uma.es (P.M.T.) \\ Correspondence: cristinags@uma.es; Tel.: +34-951-953-298 \\ †Presented at the 1st International Electronic Conference on Catalysis Sciences, 10-30 November 2020; \\ Available online: https://sciforum.net/conference/ECCS2020
}

Published: 10 November 2020.

\begin{abstract}
In this work, acidic ion-exchange resins with strong Brönsted sulphonic groups were assessed in the catalytic etherification of the platform molecule 5-(hydroxymethyl)furfural (HMF) to 5-(ethoxymethyl)furfural (EMF), a biofuel with an energy density close to that of gasoline (30 $\mathrm{MJ} / \mathrm{L}$ ) which also reduces emissions of $\mathrm{NO}_{\mathrm{x}}$ and $\mathrm{SO}_{\mathrm{x}}$ and solid particles respect to fossil-derived fuels. Catalytic performance was optimized modifying experimental parameters such as reaction time, temperature and concentration of reagent employed. This process was carried out in batch reactors using ethanol $96 \%$ as solvent. Among different cation-exchange resins tested, Purolite CT275DR provided the fastest HMF conversion together with Purolite PD206, and the highest selectivity to EMF, achieving above $70 \%$ selectivity at $100^{\circ} \mathrm{C}$. Overtime, strong acid sites favoured product hydrolysis opening the furan ring originating ethyl levulinate (EL) to the detriment of EMF selectivity. Purolite CT275DR was also utilised to realize the transformation from sugars directly to EMF in the same reaction medium, in a one-pot process, obtaining relevant results from fructose (37\% HMF yield, 21\% EMF yield after $5 \mathrm{~h}$ ), but originating selectively ethylglucosides and ethylgalactosides in the presence of glucose and galactose respectively due to the absence of necessary Lewis acid sites to isomerize aldose and proceed with dehydration.
\end{abstract}

Keywords: 5-(hydroxymethyl)furfural; 5-(ethoxymethyl)furfural; ethyl levulinate; ethanol; biofuel; monosaccharides; hexoses; etherification; ionic exchange resins

\section{Introduction}

Nowadays, fossil resource depletion as well as climate change produced by their exploitation are major concerns [1]. Biomass is receiving increasing attention due to its renewable nature, abundance and worldwide availability. Lignocellulose is the most abundant renewable source [2], since it appears in nature with structural function in plants and constitutes an important part of their dry weight. Approximately a $45 \%$ of lignocellulosic biomass corresponds to cellulose, $27.5 \%$ to hemicellulose and $17.5 \%$ to lignin [3]. Cellulose is the most abundant biopolymer in earth, and this makes glucose, its only monomer, a key compound for valorisation processes into high value-added chemicals and fuels.

Hexose triple dehydration in the presence of Brönsted acid leads to the formation of HMF, a versatile platform molecule with applications in polymer science and biofuel production [4]. EMF is 
a potential biofuel derived from HMF, which is produced from the simple etherification with ethanol of the hydroxyl group present in the HMF molecule. EMF is a substance with similar properties to those of common fuels currently employed. It has an energy density of $30.3 \mathrm{MJ} / \mathrm{L}(94 \%$ of that of gasoline) [5], improves kinematic properties in cold and reduces particle formation, $\mathrm{NO}_{\mathrm{x}}$ and $\mathrm{SO}_{\mathrm{x}}$ production respect to common fuels [6].

Under acidic media, saccharides and its valuable derivatives can produce a wide range a byproducts, as well as soluble oligomers and insoluble polymers (humins) that reduce selectivity [7-9]. EMF synthesis has been reported by using ionic liquids [10], heteropoly acids [11], ion exchange resins [6], or metal salts [12] accompanied of Lewis acid sites to promote isomerization of glucose to fructose when it was required $[6,13]$. However, difficulties remain still in the transformation of fructose to EMF, or specially starting from glucose.

Aiming to improve results and achieve more selective production from different sources, this work evaluates the behaviour of several ion exchange resins in the catalytic etherification of HMF to EMF, including the one-pot transformation of saccharides. Thus, cation exchange resins with different nature, both macroreticular (Purolite CT275DR, CT269DR, and Amberlyst 15) and a gel type resin (Purolite PD206), were tested for EMF production.

\section{Materials and Methods}

Catalysts employed were commercially available ion exchange resins. Purolite CT275DR, Purolite CT269DR and Purolite PD206 were acquired from Purolite ${ }^{\circledR}$, Amberlyst 15 from Fluka ${ }^{\circledR}$.

Etherification reactions were carried out in batch reactors (Ace pressure $15 \mathrm{~mL}$ ). Typical conditions employed were $5 \mathrm{~mL}$ of solvent (ethanol 96\%, VWR Chemicals), $0.1 \mathrm{~g}$ of HMF (99,9\%, Sigma-Aldrich), $0.05 \mathrm{~g}$ of catalysts and $100^{\circ} \mathrm{C}$, unless otherwise specified. Reactors were purged before reaction with $\mathrm{N}_{2}$ to avoid secondary reactions related to $\mathrm{O}_{2}$ presence and heated under magnetic stirring in a temperature regulated aluminium block. Reaction time started counting once it was submerged in the aluminium block, previously heated at the desired temperature. Once reaction time finished, the reactor was extracted from the aluminium block and cooled with water. For the analysis, samples were diluted in water and analysed through a JASCO high performance liquid chromatography (HPLC). The instrument employed a Phenomenex REZEX $\mathrm{Ca}^{2+}$ Monosaccharide $\left(300 \mathrm{~mm} \times 7.8 \mathrm{~mm}\right.$ ) column heated in a column oven (CO-2065) at $70^{\circ} \mathrm{C}$ with 0.4 $\mathrm{ml} / \mathrm{min}$ of deionized water flow provided by a quaternary gradient pump (PU-2089). The instrument is equipped with a multiwavelength detector (MD-2015) as well as a refractive index detector (RI2031-PLUS) for qualitative and quantitative measurement.

$$
\begin{aligned}
& \text { Conversion } \left._{\mathrm{HMF}}=\left(\left(\mathrm{mol}_{\text {initialHMF }}-\mathrm{mol}_{\text {finalHMF }}\right)\right) / \mathrm{mol}_{\text {initialHMF }}\right) \cdot 100 \\
& \text { Selectivity }_{\mathrm{X}}=\left(\mathrm{mol}_{\text {finalX }} /\left(\mathrm{mol}_{\text {initialHMF }_{\text {MF }}}-\operatorname{mol}_{\text {initialHMF }}\right)\right) \cdot 100 \\
& \text { Yield }_{\mathrm{X}}=\left(\text { Selectivity }_{\mathrm{X}} \cdot \text { Conversion }_{\mathrm{HMF}}\right) / 100
\end{aligned}
$$

\section{Results and Discussion}

Firstly, these cation exchange resins were submitted to catalytic screening under aforementioned conditions $\left(100^{\circ} \mathrm{C}, 0.1 \mathrm{~g} \mathrm{HMF}, 0.05 \mathrm{~g}\right.$ catalyst, $5 \mathrm{~mL}$ ethanol) at different reaction times (Figure $\left.1 \mathrm{a}\right)$. HMF conversion followed a first order reaction (Figure 1 b) with very high conversion values after 24 hours of reaction for all studied resins. Purolite CT275DR and Purolite PD206 seemed to be slightly more active than the others. Regarding the selectivity, resins could produce EMF with selectivities above 70\%, concretely Purolite CT275DR in a time below $9 \mathrm{~h}$ or Purolite CT269DR after $9 \mathrm{~h}$ reaction. EMF selectivity values become increasingly worse as time progresses, decreasing due to secondary reactions (Figure 1c). Therefore, reaction time is a factor that affects unfavorably EMF selectivity. HMF and EMF rehydration are known to produce levulinic and formic acids, or their respective esters in alcohol media [14]. Over time, EL selectivity increased in agreement with this statement (Figure 
1d). While Purolite CT275DR, Purolite CT269DR and Amberlyst 15 presented similar values of EL selectivity at all times, Purolite PD206 particularly showed the highest EL selectivity among the resins studied, up to $26 \%$, after $24 \mathrm{~h}$. It should be noted that purolites provided the best selectivity results towards EMF surpassing that of Amberlyst 15.

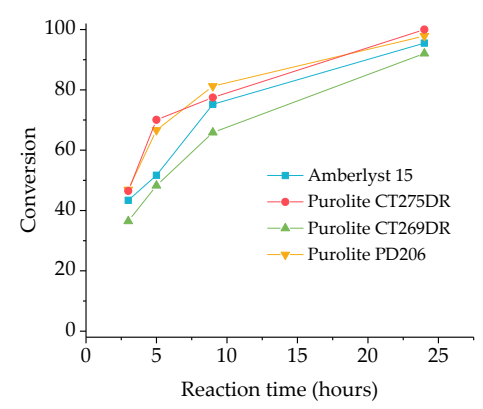

(a)

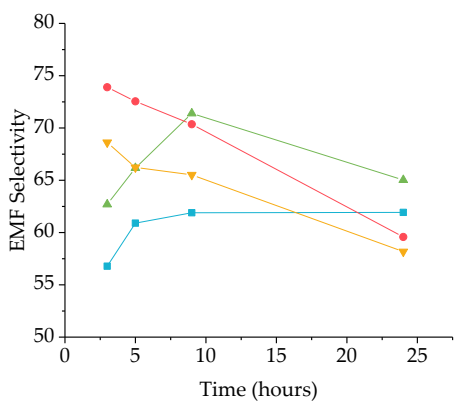

(c)

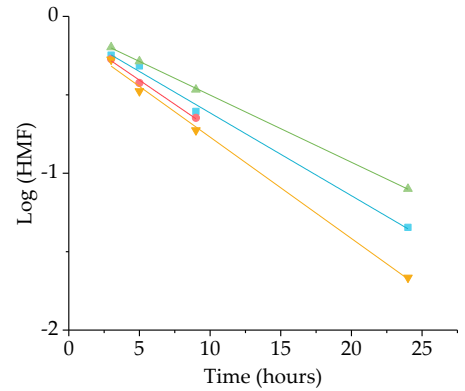

(b)

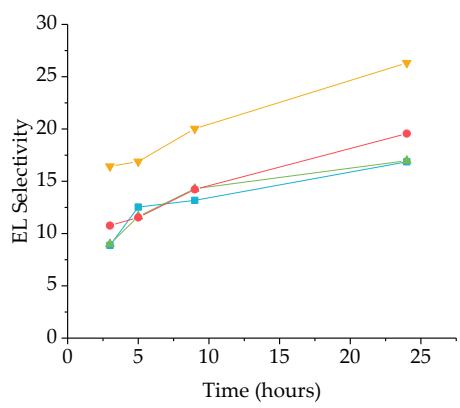

(d)

Figure 1. (a) Conversion of $\mathrm{HMF}$ at $100^{\circ} \mathrm{C}$. (b) Logarithmic representation of HMF concentration respect to time. (c) Measured selectivity towards EMF production. (d) Measured selectivity towards EL formation. ( $100^{\circ} \mathrm{C}, 0.1 \mathrm{~g} \mathrm{HMF}, 0.05 \mathrm{~g}$ catalyst, $5 \mathrm{~mL}$ ethanol at different reaction time).

However, it is important to attain the direct transformation of saccharides to EMF instead of utilizing HMF as feedstock, given that these saccharides can be easily accessible from lignocellulose. Hence, Purolite CT275DR was tested by using fructose, glucose and galactose to obtain EMF in a onepot reaction (Figure 2). Fructose was readily dehydrated in the presence of strong Brönsted acid sites present on the resin, giving rise to a HMF yield of $39 \%$ after $5 \mathrm{~h}$. Moreover, EMF was detected from fructose, so HMF was etherified to EMF, obtaining a EMF yield of $20 \%$ after $5 \mathrm{~h}$. Nevertheless, aldoses need the presence of Lewis acid sites to isomerize to ketoses and proceeds with dehydration to form HMF. As expected, neither glucose nor galactose produced HMF, EMF or EL. Both reagents were transformed selectively to a single unidentified product, which, according to previous works dealing with the treatment of glucose with Brönsted acidic solids in the presence of ethanol [11], could be ethyl glucoside and ethyl galactoside, respectively. 


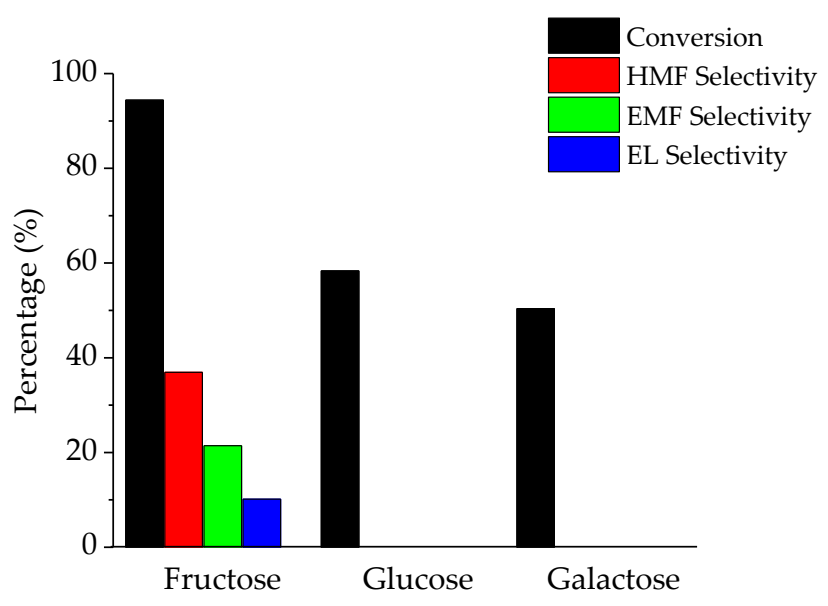

Figure 2. Conversion and selectivities towards HMF, EMF and EL $\left(100^{\circ} \mathrm{C}, 0.15 \mathrm{~g}\right.$ hexose, $0.05 \mathrm{~g}$ Purolite CT275DR, 5mL ethanol for 5 hours).

\section{Conclusions}

Several resins were assessed in the etherification reaction of HMF to EMF by using ethanol as solvent. The resins showed relevant conversion values. The use of purolite resins resulted in good values of EMF selectivity, particularly the use of Purolite CT275DR, which also showed the best conversion values together with Purolite PD206. To prove the applicability of the resin to saccharide conversion, fructose, glucose and galactose were employed as feedstock, under similar experimental conditions instead of HMF, as reagent. As expected, the resin failed to convert satisfactorily aldoses to HMF in the absence of Lewis acid catalyst, but Purolite CT275DR successfully transformed fructose with a HMF yield of $39 \%$ and an EMF yield of $20 \%$ after 5 hours.

Author Contributions: conceptualization, P.M.T. and C.G.S; methodology, C.G.S; formal analysis, B.T.O.; investigation, B.T.O. and I.F.Ñ.; resources, C.G.S.; data curation, B.T.O., C.G.S and I.F.Ñ.; writing - original draft preparation, B.T.O., C.G.S and J.A.C.; writing - review and editing, C.G.S., J.A.C., R.M.T. and P.M.T.; project administration, C.G.S. and P.M.T.; funding acquisition, C.G.S., R.M.T. and P.M.T.

Funding: This research was funded by the Spanish Ministry of Economy and Competitiveness (RTI2018-94918B-C44), FEDER (European Union) funds (UMA18-FEDERJA-171) and Malaga University.

Acknowledgments: J.A.C. thank to the Malaga University for the financial support. C.G.S. acknowledges FEDER funds for a postdoctoral contract (UMA18-FEDERJA-171).

Conflicts of Interest: The authors declare no conflict of interest.

\section{References}

1. IPCC Global warming of $1.5^{\circ} \mathrm{C}$; V. Masson-Delmotte, P. Zhai, H. O. Pörtner, D. Roberts, J. Skea, P.R.S., A. Pirani, W. Moufouma-Okia, C. Péan, R. Pidcock, S. Connors, J. B. R. Matthews, Y. Chen, X. Zhou, M.I.G., E. Lonnoy, T. Maycock, M. Tignor, T.W., Eds.; Geneva, Switzerland, 2018;

2. Zhou, C.H.; Xia, X.; Lin, C.X.; Tong, D.S.; Beltramini, J. Catalytic conversion of lignocellulosic biomass to fine chemicals and fuels. Chem. Soc. Rev. 2011, 40, 5588-5617.

3. Menon, V.; Rao, M. Trends in bioconversion of lignocellulose: Biofuels, platform chemicals \& biorefinery concept. Prog. Energy Combust. Sci. 2012, 38, 522-550.

4. Mika, L.T.; Cséfalvay, E.; Németh, Á. Catalytic Conversion of Carbohydrates to Initial Platform Chemicals: Chemistry and Sustainability. Chem. Rev. 2018, 118, 505-613.

5. Liu, X.; Wang, R. Upgrading of Carbohydrates to the Biofuel Candidate 5-Ethoxymethylfurfural (EMF). Int. J. Chem. Eng. 2018, 2018.

6. Lew, C.M.; Rajabbeigi, N.; Tsapatsis, M. One-pot synthesis of 5-(Ethoxymethyl)furfural from glucose using Sn-BEA and Amberlyst catalysts. Ind. Eng. Chem. Res. 2012, 51, 5364-5366. 
7. Holm, M.S.; Saravanamurugan, S.; Taarning, E. Conversion of Sugars to Lactic Acid Derivatives Using Heterogeneous Zeotype Catalysts. Science (80-. ). 2010, 328, 602-605.

8. Zhang, J.; Weitz, E. An in situ NMR study of the mechanism for the catalytic conversion of fructose to 5Hydroxymethylfurfural and then to levulinic acid using 13c labeled d -fructose. ACS Catal. 2012, 2, 12111218.

9. van Putten, R.-J.; van der Waal, J.C.; de Jong, E.; Rasrendra, C.B.; Heeres, H.J.; de Vries, J.G. Hydroxymethylfurfural, A Versatile Platform Chemical Made from Renewable Resources. Chem. Rev. 2013, 113, 1499-1597.

10. Guo, H.; Duereh, A.; Hiraga, Y.; Qi, X.; Smith, R.L. Mechanism of Glucose Conversion into 5Ethoxymethylfurfural in Ethanol with Hydrogen Sulfate Ionic Liquid Additives and a Lewis Acid Catalyst. Energy and Fuels 2018, 32, 8411-8419.

11. Yang, Y.; Abu-Omar, M.M.; Hu, C. Heteropolyacid catalyzed conversion of fructose, sucrose, and inulin to 5-ethoxymethylfurfural, a liquid biofuel candidate. Appl. Energy 2012, 99, 80-84.

12. Liu, B.; Zhang, Z.; Huang, K.; Fang, Z. Efficient conversion of carbohydrates into 5-ethoxymethylfurfural in ethanol catalyzed by $\mathrm{AlCl}$. Fuel 2013, 113, 625-631.

13. Mahmoud, E. Glucose Conversion to Furans in Alcohols Catalyzed by Lewis Acidic Beta Zeolites and Brønsted Acidic Resins. ChemistrySelect 2017, 2, 10336-10339.

14. Flannelly, T.; Dooley, S.; Leahy, J.J. Reaction Pathway Analysis of Ethyl Levulinate and 5Ethoxymethylfurfural from d -Fructose Acid Hydrolysis in Ethanol. Energy and Fuels 2015, 29, 7554-7565.

Publisher's Note: MDPI stays neutral with regard to jurisdictional claims in published maps and institutional affiliations.

(C) 2020 by the authors. Submitted for possible open access publication under the terms and conditions of the Creative Commons Attribution (CC BY) license (http://creativecommons.org/licenses/by/4.0/). 